\title{
Application of mathematics to flow in porous media before the computer age; an introduction to the Special Issue "Applying mathematics to flow in porous media"
}

\author{
O. D. L. Strack - Anvar R. Kacimov
}

Received: 13 March 2009 / Accepted: 13 March 2009 / Published online: 31 March 2009

(C) Springer Science+Business Media B.V. 2009

"Porous-media flow" is a generalization of the older and more common term "groundwater flow"; it removes the limitation of the field to the flow of water through the ground to include the flow of water through any porous substance. A similar generalization took place when the term "consolidation" became "poro-elasticity".

This Special Issue of the Journal of Engineering Mathematics deals with the application of mathematics to porous-media flow and poro-elasticity. The latter field could be considered to contain the first as a special case: poro-elasticity is concerned with the flow of a fluid through an elastic medium with full consideration of the stress and displacement fields; it reduces to flow through porous media if the vertical total stress in the porous medium is a constant.

This special issue contains nine papers, selected for their application of mathematics. The nine papers are grouped according to their subject matter. The first two papers are concerned with steady flow, the third and fourth ones with transient flow, and the fifth and sixth ones with multi-aquifer flow. The seventh and eighth papers deal with unsaturated flow, and the final paper with poro-elasticity.

Steady groundwater flow in a homogeneous porous medium is governed by Laplace's equation; compared to other fields in engineering and geophysics, its governing equation has a rich collection of elegant solutions as well as methods of solution, particularly in two dimensions. The difficulty in solving groundwater flow problems stems not from the complexity of the governing equations, but rather from the enormous variety of boundary conditions that occur in natural groundwater environments; such a situation invites the application of mathematics to solve practical problems. Today, the use of complex computer models is common for solving problems in porous-media flow, often coupled with other phenomena such as heat conduction, contaminant transport, and chemical or biological processes. Historically, however, the field has been dominated by the derivation and application of closed-form analytical solutions.

We attempt to give a brief overview of this history to introduce the reader to the rich collection of elegant mathematical techniques and solutions in the field of groundwater flow. The emphasis shifted over the years from the development of elegant analytical solutions to the reliance on existing computer programs to solve practical problems. This shift came with the arrival of the computer age, which led to the development of computer packages

O. D. L. Strack $(\bowtie)$

Department of Civil Engineering, University of Minnesota, 500 Pillsbury Dr. S.E, Minneapolis, MN 55455, USA

e-mail: strac001@umn.edu

A. R. Kacimov

Sultan Qaboos University, Muscat, Sultanate of Oman 
based on discrete numerical methods. We limit this brief overview to developments prior to the computer age, which began in earnest around the middle 1960s.

The field of groundwater flow changed from an art to a quantitative science with the establishment of the basic law of groundwater flow discovered by Henry Darcy (1803-1858) in 1856, called Darcy's law [1]. This law relates the specific discharge vector with components $q_{x}, q_{y}, q_{z}$ [L/T] in three dimensions to the hydraulic conductivity, $k$ $[\mathrm{L} / \mathrm{T}]$ and the piezometric head $h[\mathrm{~L}]$ as follows, for isotropic hydraulic conductivity:

$$
\begin{aligned}
& q_{x}=-k \frac{\partial h}{\partial x} \\
& q_{y}=-k \frac{\partial h}{\partial y} \\
& q_{z}=-k \frac{\partial h}{\partial z}
\end{aligned}
$$

This equation, combined with the mass balance equation, which is, for incompressible flow,

$\frac{\partial q_{x}}{\partial x}+\frac{\partial q_{y}}{\partial y}+\frac{\partial q_{z}}{\partial z}=0$

yields, for constant $k$, Laplace's equation,

$\nabla^{2} h=0$

We remarked that groundwater flow problems are characterized by difficult boundary conditions; this becomes apparent even when considering the relatively simple problem of unconfined flow in a water-bearing layer, or aquifer (from Latin: aqua (water) + fero (to carry)). The free-boundary condition along the water table, or phreatic surface, is complicated, and a method of solution of such problems had yet to be devised. In addition, exact solutions to such problems are limited to cases of two-dimensional flow in the vertical plane, or radial flow in the vertical plane. Thus, solving practical problems governed by Darcy's law and the mass balance equation was not possible, even for the most elementary problems of groundwater flow.

The next great step in the development of groundwater flow as a science was due to Jules Dupuit (1804-1866); see [2]. He assumed that the piezometric head in the aquifer, even with groundwater moving, is hydrostatic, i.e., that the vertical variation of the horizontal components of the specific discharge vector can be ignored. This approximation, similar to the shallow-water theory in fluid mechanics, has proven to be far better than one might expect, does not restrict the flow to be horizontal (as is often assumed), and yields the exact discharge flowing through a section of aquifer bounded by two parallel boundaries of fixed head. Interestingly, this simplification was assumed to yield only approximate discharges, and studies were carried out to estimate the error in the so-called Dupuit formula,

$Q_{x}=k \frac{h_{1}^{2}-h_{2}^{2}}{2 L}$

where $h_{1}$ and $h_{2}$ are the piezometric heads along the two parallel boundaries, and $Q_{x}$ is the discharge $\left[\mathrm{L}^{2} / \mathrm{T}\right]$ flowing through the aquifer; the piezometric heads are measured relative to the horizontal base of the aquifer. A similar equation applies to radial flow toward a well; in that case the dependence of the discharge on the differences in the squares of the heads involves the logarithm of the distance. A laboratory study carried out by Wyckoff et al. [3] was aimed at establishing the error in the Dupuit formula. As we now know, this error is zero; the laboratory study confirmed this. It was not until 1951 that Charney [4] proved mathematically that Dupuit's formula is exact, i.e., it yields precisely the same discharge as would be obtained by solving the boundary-value problem in terms of Laplace's equation in three dimensions. This remains true in far more general settings than expected; see [5]. The approximation made by Dupuit is often referred to as the Dupuit-Forchheimer approximation, in honor of the Austrian engineer Philip Forchheimer (1852-1933), see [6], who made the approximation independently of Dupuit in similar context. According to the Encyclopedia Brittanica, Forchheimer [7] was the first to associate Laplace's equation with groundwater flow, thereby opening a wealth of existing solutions to the field. In Russia, Zhukovsky [8] pioneered the derivation of the equations of groundwater motion from the Navier-Stokes equations, also arriving 
at Laplace's equation for incompressible flow in porous media. Pavlovsky [9] studied flow of groundwater underneath hydraulic structures, and emphasized that "the motion of groundwater should be considered as a problem of mathematical physics", which he referred to as "the most important problems of engineering design in the USA, as a country of the most advanced and practical geotechnical engineering".

A class of problems of considerable practical importance consists of those with a free boundary. Zhukovski [10] developed a complex function, now known as the Zhukovski function, that has constant real or imaginary parts along certain boundaries, in particular boundaries that involve a free surface. A problem of importance for examining the stability of dams is that of free-surface flow toward a horizontal drain. This problem was first solved by Vreedenburgh [11], but published in Dutch and little known outside of that country, and independently by Pavlovsky [12]. A more general approach to solving problems with a free boundary is based on the hodograph method, well known in fluid mechanics. This method was first applied in the field of groundwater flow by Hamel [13]. He considered the problem of flow through a dam with vertical faces and constructed the corresponding domain in the hodograph plane. Vedernikov [14] derived outstanding analytical solutions for Darcian flows in application to irrigation and drainage problems.

Studies of porous-media flow in the Soviet Union had a sound mathematical basis and three Soviet scientists, Polubarinova-Kochina, Numerov, and Charny contributed significantly to the many elegant analytical solutions in the field of groundwater mechanics. The textbooks written by Polubarinova-Kochina $[15,16]$ and Aravin and Numerov $[17,18]$ are considered classics in the field of groundwater flow. Unfortunately, other significant textbooks, $[19,20]$, are written in Russian, limiting the accessibility of the solutions presented in these books to those who master the Russian language.

Yet another class of groundwater flow problems concerns flow in coastal aquifers; an important class of problems in many countries, e.g., the USA, The Netherlands, and Israel, to name but a few. A fundamental formula that deals with fresh-water salt-water interfaces is the Ghyben-Herzberg formula, due to independent discoveries by Badon-Ghyben [21] and Herzberg [22]. The formula relates the head in the fresh-water and salt-water zones assuming a hydrostatic pressure distribution and requiring that the pressures are equal on both sides of the interface between fresh water and salt water. The combination of this formula with the Dupuit-Forchheimer approximation makes it possible to obtain useful solutions to problems of groundwater flow in coastal aquifers.

Wells were increasingly used for groundwater recovery, and the original solutions were generalized; the Thiem solution for steady flow toward a well [23], and the solution for transient flow toward a well proposed by Theis [24]. Extension to flow in compressible aquifers and the study of leakage between aquifers is due to Jacob [25]. Hantush [26] made many contributions, often working with Jacob, on the application of mathematics to transient groundwater flow and flow in leaky aquifers.

An elegant set of solutions to Laplace's equations, applicable to problems of groundwater flow in the vertical plane with a phreatic surface and infiltration, was developed by Toth [27], which is considered a landmark analysis in that it attempts to relate topography to groundwater flow.

Although poro-elasticity is often not considered part of porous-media flow, the relationship is sufficiently close to present some of the background. Poro-elasticity was originally called consolidation theory; the first formulation is due to Karl von Terzaghi, considered to be the father of soil mechanics [28], who developed an equation that includes the change of storage of the fluid due to compression of the medium, which is still in use in the field of groundwater flow today. However, Terzaghi's approach is correct only if the total vertical stress in the medium is constant in time and in space, which is a good approximation in many cases of groundwater flow, but not in soil mechanics, where the total vertical stress may change substantially due to the addition of a load on the surface. A complete theory of poro-elasticity was first presented by Biot [29].

When computers became widely available in the nineteen-seventies, the need for constructing mathematically elegant solutions was reduced; the belief became increasingly common that relatively simple discretized computer models of groundwater flow are the best way to solve practical problems, and that working on analytical solutions was essentially a waste of time. This belief is flawed for two reasons. The first is that analytic solutions and an understanding of the mathematical foundation are prerequisites for an understanding of engineering problems. Even if these analytical solutions merely provide a first rough estimate of the answer to a practical problem, such solutions 
invariably increase the understanding of the problem and its controlling parameters. The second flaw is that the belief that analytical representations are incapable of the generality that is required to solve real-life problems is false. We hope that this Special Issue goes some way to demonstrate that analytical solutions are by no means obsolete; it may, in fact, turn out that the development and use of closed-form analytical expressions is still in its infancy.

One might ask the question why to pursue elegant mathematical solutions at a time when computational power seems to indicate that this is no longer necessary. As an answer, consider the following quote by Poincare (18541912) [30]: "What is it indeed that gives us the feeling of elegance in a solution, in a demonstration? It is the harmony of the diverse parts, their symmetry, their happy balance; in a word it is all that introduces order, all that gives unity, that permits us to see clearly and to comprehend at once both the ensemble and the details". In closing, it may be of interest to add that several papers in this special issue make use of the non-Cartesian complex $(z, \bar{z})$ space, an idea first introduced by Poincaré, and now proving to be of highly practical use; applications that could not be contemplated without access to highly efficient computational engines with complex functions built right into the chips.

\section{References}

1. Darcy H (1856) Les Fountaines Publiques de la Ville de Dijon. Dalmont, Paris

2. Dupuit J (1863) Études Théoriques et Pratiques sur le Mouvement des Eaux dans les Canaux Decouverts et à Travers les Terrains Perméables, 2íeme ed. Dunod, Paris

3. Wyckoff RD, Botset HG, Muskat M (1932) Flow of liquids through porous media under the action of gravity. Physics 3:90-113

4. Charny IA (1951) A rigorous derivation of Dupuit's formula for unconfined seepage with a seepage surface. Dokl Akad Nauk SSSR 79:937-940

5. Strack ODL, Barnes RJ, Verruijt A (2005) Vertically integrated flows and the Dupuit-Forchheimer approximation. Ground Water 44(1):72-75

6. Forchheimer P (1886) Ueber die Ergiebigkeit von Brunnen- Anlagen und Sickerschlitzen. Z Architkt Ing Verlag 32:539-563

7. Forchheimer P (1914) Hydraulik. B.G. Teubner, Leipzig

8. Zhukovsky NE (1889/1949) Theoretical investications about the movement of subterranean water. In: Collected works, vol 5, pp 184-206. Gostekhizdat, Moscow

9. Pavlovski NN (1922) The theory of groundwater flow under hydraulic structures. Lithographic, Petrograd

10. Zhukovski NE (1920/1950) Seepage through dams. In: Collected works. vol V, pp 297-332. Gostekhizdat, Moskow

11. Vreedenburgh CGJ (1929) Over de vlakke stationnaire waterstoming door een homogene grondmassa, onder aanname van twee stelsels confocale parabolen als stroom- en potentiaal lijnen. TH, Bandoeng

12. Pavlovski NN (1937) About the flow of water to an horizontal drain. Izv. NIIG, 21

13. Hamel G (1934) Ueber Grundwasser. Z Angew Math Mech 14(3):129-157

14. Vedernikov VV (1939) Seepage theory and its application in the field of irrigation and drainage. State Press, Moscow

15. Polubarinova-Kochina PY (1952) Theory of groundwater movement (in Russian). Gostekhizdat, Moskow

16. Polubarinova-Kochina PY (1962) Theory of groundwater movement. Princeton University Press, Princeton, NJ

17. Aravin VI, Numerov SN (1953) Theory of motion of liquids and gases in undeformable porous media. Israel Program for Scientific Translation, Jerusalem

18. Aravin VI, Numerov SN (1965) Theory of fluid flow in undeformable porous media. Daniel Davey, New York

19. Polubarinova-Kochina PY (1977) Theory of groundwater movement. Nauka, Moscow

20. Charny IA (1963) Subsurface hydro-gas-dynamics. GGNTINGTL, Moscow

21. Badon-Ghyben W (1888-1889) Nota in verband met de voorgenomen put boring nabij Amsterdam. Tijdschr Kon Inst Ing, The Hague, pp 8-22

22. Herzberg A (1901) Die Wasserversorgung einiger Nordseebaden. Z Gasbeleucht Wasserverzorg 44:815-819, 824-844

23. Thiem G (1906) Hydrologische Methoden. J. M. Gebhardt, Leipzig, Germany

24. Theis CV (1935) The relation between lowering of the piezometric surface and the rate and duration of the discharge of a well using ground water storage. In Transactions American Geophysical Union, 16th meeting. vol 2, pp 519-524

25. Jacob CE (1940) The flow of water in an elastic artesian aquifer. Trans Am Geophys Union 21:574-586

26. Hantush MS (1964) Advances in hydroscience. I: Chapter hydraulics of wells. Academic Press, New York

27. Toth J (1963) A theroretical analysis of groundwater flow in small drainage basins. J Geophys Res 68(16):4795-4812

28. von Terzaghi K (1925) Erdbaumechanik. Franz Deuticke, Vienna

29. Biot MA (1941) General theory of three-dimensional consolidation. J Appl Phys 5:339-404

30. Poincaré J-H (1908) Science et Méthode 